\title{
Advances in neutronics and radiological protection of HiPER 4a
}

\author{
Rafael Juárez ， Javier Sanz ， José M. Perlado
}

\begin{abstract}
A B S T R A C T
The HiPER project, phase 4a, is evolving. In this study we present the progress made in the field of neutronics and radiological protection for an integrated design of the facility. In the current model, we take into account the optical systems inside the target bay, as well as the remote handling requirements and related infrastructure, together with different shields. The last reference irradiation scenario, consisting of $20 \mathrm{MJ}$ of neutron yields, 5 yields per burst, one burst every week and 30 years of expected lifetime is considered for this study. We have performed a characterization of the dose rates behavior in the facility, both during operation and between bursts. The dose rates are computed for workers, regarding to maintenance and handling, and also for optical systems, regarding to damage. Furthermore, we have performed a waste management assessment of all the components inside the target bay. Results indicate that remote maintenance is mandatory in some areas. The small beam penetrations in the shields are responsible for some high doses in some specific locations. With regards to optics, the residual doses are as high as prompt doses. It is found that the whole target bay may be fully managed as a waste in 30 years by recycling and/or clearance, with no need for burial.
\end{abstract}

\section{Introduction}

It is foreseen that High Power laser Energy Research (HiPER) phase $4 a$ will be an experimental facility for testing the integration of high repetition rate technologies related to Inertial Fusion Energy (IFE). During the last several years, the different groups inside the HiPER project have proposed alternative solutions for the systems they are in charge of (optical assembly, lasers, target injection...). A preliminary integrated design, coupling optical systems together with shields against neutrons and gammas, was evaluated from the standpoint of radiological assessment [1]. A deep study was also accomplished on the materials selection for the reaction chamber [2]. Linking the conclusions of those studies $[1,2]$ to some changes in the baseline design, a new and advanced design of HiPER 4a target bay is ready. In this advanced design three systems are integrated: optical systems, remote maintenance systems and shielding requirements. The arrangement of the systems is described in the subsection 1.1. It has been decided to set the reference irradiation scenario of HiPER 4a as follows: It will operate in 100 shots bursts. In every burst there will be only 5 ignitions, with $20 \mathrm{MJ}$ neutron yields each one. The other 95 shots, non-yield shots, will not ignite, but they will be oriented to other aspects of illumination. However, aspects such as debris or shrapnel are not addressed in this study. One burst will take place every week, what represents $5200 \mathrm{MJ} /$ year of neutron yield. It is assumed a 30 years lifetime for the facility.

We present a dose rates and waste management assessment for the advanced design of HiPER 4a target bay, considering the new reference irradiation scenario. Taking into account the obtained results, we offer an evaluation of the shielding proposal regarding to protection to workers and to optical systems during the operation of the facility. In the time between bursts, we characterize the different areas from the standpoint of residual dose rates for workers. We offer a waste management assessment of all the components inside the target bay.

\subsection{Baseline design}

The advanced design of HiPER 4a, integrates the main systems of the target bay: optical systems, remote handling infrastructure, and shielding requirements. The different systems and their configuration are briefly explained in this section. Figs. 1 and 2 are devoted to explain this design.

Centered in the origin, there is the reaction chamber. It has an inner radius of $5 \mathrm{~m}$, and $10 \mathrm{~cm}$ of thickness. It is built of T91 commercial steel [3]. The chamber presents 48 penetrations for the laser beams. The beams are brought to the chamber through 48 beam tubes which keep the vacuum inside. These tubes are also made of T91 steel. They have a squared section of $1 \mathrm{~m}$ per $1 \mathrm{~m}$. The wall thickness of the beam tubes is $1 \mathrm{~cm}$. At $8 \mathrm{~m}$ from the origin and 


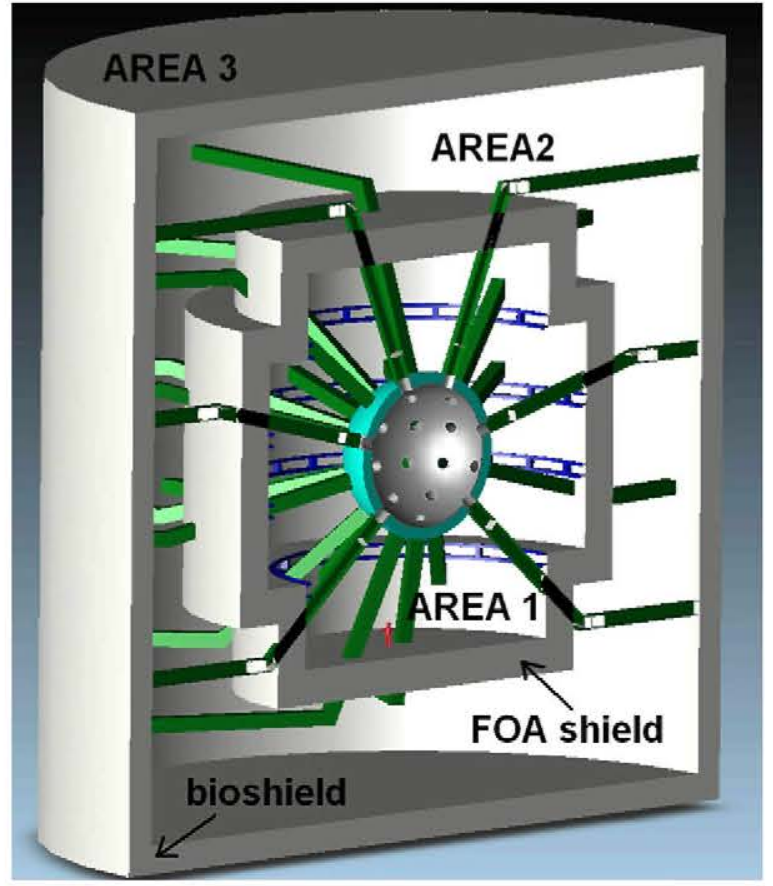

Fig. 1. General view of the advanced design of HiPER 4a.

inside the beam tubes, there are 48 focusing lenses. They are called the "renewable optics", and they are placed in front of the explosions with no shield to protect them against the resulting radiation. The renewable optics are squared lenses of $75 \mathrm{~cm}$ side and $5 \mathrm{~cm}$ of thickness. At $19 \mathrm{~m}$ from the origin, also inside beam tubes, there are 48 groups of optical elements, named the Final Optics Assembly (FOA). These groups consist of 6 optical elements with different functions (mirrors, focusing lenses and frequency converters). All the elements are squares of $75 \mathrm{~cm}$ side, with thicknesses varying from 1 to $5 \mathrm{~cm}$. They are also made of pure silica.

We have proposed four shields in the target bay to protect both the workers and the optics. The first one is placed against the reaction chamber and it is called the chamber shield. It consists of a spherical shell of $80 \mathrm{~cm}$ of thickness, made of borated concrete. This material choice is motivated by NIF [4] choice of chamber shield. Given the efficiency of shielding close to the source, its thickness has doubled from the previous design. We pursue a general reduction of neutron spreading with this decision. At $16 \mathrm{~m}$ from the origin, and outside the beam tubes, there is the FOA shield. It is a $2.5 \mathrm{~m}$ thick shield made of standard concrete. To improve the previous situation between bursts in some areas regarding waiting

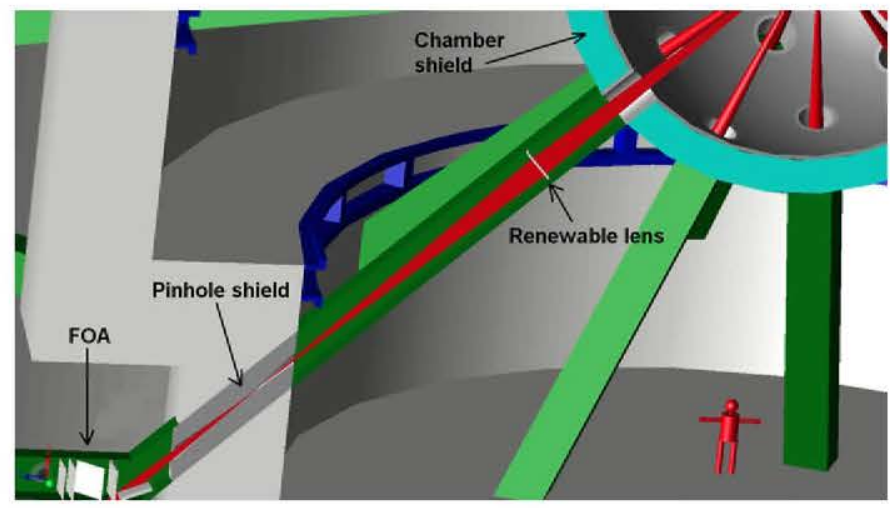

Fig. 2. Detailed view of the main optical systems: renewable lenses, final optics assembly, pinhole shield and beam tubes. time for workers entrance, its size has increased from 2 to $2.5 \mathrm{~m}$ from the previous design, and its shape has changed from spherical to a double cylinder, because of remote handling systems requirements. At the same position, but inside the beam tubes, there are the pinhole shields. They are 48 concrete cylinders, with $50 \mathrm{~cm}$ of radius and $2.5 \mathrm{~m}$ long, placed inside the beam tubes, presenting a conical perforation to allow the beam go through. Finally, there is a cylindrical shell shape bioshield of $25 \mathrm{~m}$ of inner radius and $2 \mathrm{~m}$ of thickness. This shield has not been optimized, as beam penetrations sizes have not been defined and a lot of machinery has still to be defined. However, it is expected that this bioshield will be the final barrier against radiation during the operation of the facility. This distribution of shields defines 4 areas which need a radiological classification with regards to workers access both during operation and during the maintenance. The interior of the chamber is well defined, and requires no special name. The space between the chamber shield and the FOA shield is called area 1. The space between the FOA shield and the bioshield is called the area 2 ; the space outside the bioshield is called area 3 . We assume only workers in the facility, thus no reference is made to public.

The remote handling systems requirements have forced the FOA shield to change its shape from spherical to double cylinder. Attached to this shield, there are four rails, from which the robots will access to the beam tubes, renewable optics and to outer part of the reaction chamber. The rails are made of steel alloy BS970$817 \mathrm{M} 40$ [5]. The remote maintenance in the interior of the reaction chamber does not impose restrictions on the shields, but on the chamber material. As it will be necessary for the chamber to exert some structural functions, aluminum alloys are definitively abandoned as candidate materials for the reaction chamber in benefit of steel alloys. Commercial T91 steel has been selected from a previous study [2] for waste management considerations.

\subsection{Objectives}

We present a dose rate and waste management assessment of the advanced design of HiPER 4a target bay, integrating optical systems, remote handling infrastructure and shielding requirements. We pursue to characterize the radiological performance and justify the materials choice for this design. We divide the study in three stages of the lifetime of the facility: burst operation, period between bursts, and decommissioning. This study is interesting to make decisions on many aspects, such as materials choice, placement of equipments, systems configurations or remote maintenance design.

\section{Study scope}

The radiation presence offers a natural division in three stages in the time of interest for this study: the burst operation of the facility, where important neutron and gamma dose rates levels are reached during short periods of time; the time between bursts, where gamma dose rates resulting from the activation of the facility can be high enough to preclude the access of worker to certain areas; and the decommissioning, the period after the definitive shutdown of the facility when cooling times are spent for different waste management strategies for the activated components. All the materials have been studied considering a real or reasonable impurities concentrations, and their effect on the activation behavior has been taken into account.

\subsection{Burst operation}

While explosions happen, there is a neutron spreading. A lot of these neutrons interact and generates gamma rays, which also spread through the target bay. The combination of neutron and 
gamma fluxes gives rise to high dose rates which affect to the workers and to the optical systems. Regarding to the workers, we compute the ambient dose equivalent [6], and take into consideration the limit for workers $20 \mathrm{mSv} /$ year [7]. We have performed a dose rate map of the target bay. With regards to the optical elements, we compute the absorbed dose, via the deposited energy, by neutrons and gamma separately, in the renewable lenses and in the FOA.

The dose rates map during operation offers a radiological evaluation of the different rooms inside the target bay. This map is useful for several reasons. We assign different classifications to rooms where the dose rates are above the limit during operation to the areas where workers could stand. An idea about the cold and hot regions inside every room is obtained. For example, if certain equipment may remain during the explosions in any area, where to place it is an important information. This map represents an evaluation of the proposal of shielding. If necessary, further modifications to the shielding scheme will be made if, for example, better shielding is necessary for the optical systems, or electronic devices are defined to be placed in hot areas. The damage to optical systems induced by the irradiation is a complex phenomenon requiring a study apart. The group called "renewable lenses" is troublesome in this design, and its survivability is not clear due to the impact of ions and X-rays. However, the FOA is properly protected against short penetration radiation, and its survivability is assumed. The important damage for the FOA is induced by the neutrons and gammas. We assess the delivered dose rates to FOA during the burst operation. We compare that value against what would be obtained in the case of absence of FOA and pinhole shield. This comparison stands for an evaluation of shields.

To do these calculations, we model and translate the geometry with MCAM [8]. It is a computational tool that performs 3D design, and generates an input for the geometry for MCNPX [9]. We use MCNPX to transport the neutrons and the gamma. With the flux-to-dose conversion coefficients [6] we obtain the ambient dose equivalent, and express it in a dose map of the facility. Also with MCNPX we compute directly the deposited energy in the optical systems. To perform the particles transport, we have used the ENDF-VII [10] neutron transport data library.

\subsection{Period between bursts}

Due to the neutron spreading during the explosions, the components inside the target bay get activated. The resulting gamma irradiation gives rise to residual dose rates. These doses may affect to the workers and optical elements. Regarding to the workers, in the period between bursts, the manual maintenance is not possible in some areas. As no specific task has yet been defined, the computed residual dose rates will be averaged on the whole space between shields. For workers we compute the ambient dose equivalent. With regards to the optics, the absorbed dose on the elements is calculated. For both calculations, it is also studied the partial contribution of the different elements to the residual dose rate, to identify potential responsible components. This information could be useful to reduce the residual dose rate by acting the specific troublesome components.

From the point of view of computational methodology, this calculation is performed in several steps. First of all, the neutron flux is computed in every single component of the target bay. With MCNPX, this information is requested, in a Vitamin-J structure valid for using EAF-2010 activation libraries [11]. The neutron fluxes together with the EAF-2010 libraries are used by ACAB code [12] to evaluate the isotopic inventory and its time evolution. The resulting decay gammas computed with ACAB are introduced in MCNPX. The last step consists of the transport of the resulting gamma irradiation to evaluate the ambient dose equivalent averaged in the areas between shields, and the absorbed dose in the FOA.

\subsection{Decommissioning}

It is expected that after 30 years of operation, the facility had completed its lifetime. The components inside the target bay that have been exposed to neutron irradiation may have become radioactive and require a special consideration as wastes. We perform a waste management assessment (WMA) of all the components inside the target bay to support the decision making on the choice of materials and also to estimate and show general trends about the management of the activated material. This study of the WMA is the continuity of [2], where we evaluated the behavior of different steels for the reaction chamber.

In the evaluation of the waste management, we have considered the following strategies: clearance, recycling and burial, with this order of preference. We mainly focus on clearance as the most attractive approach to the problem. If clearance is not possible, we evaluate the recycling possibility as alternative to clearance for waste management. The clearance is allowed when the clearance index (CI) is below unity [13]. If Clearance is not possible, recycling is the second best approach. Depending on the material, the criteria for recycling are the specific activity or the decay heat. If the material is a steel alloy, the adopted upper limit for recycling is $1000 \mathrm{~Bq} / \mathrm{g}$ [14]. Above this limit, steels may not be melted in foundries. If steel alloy is not the material to be recycled, the decay heat seems to be the only limitation for recycling. Thus, we adopt $2000 \mathrm{~W} / \mathrm{m}^{3}$ [14], the active cooling limit as the upper limit for recycling. However it could be the case that none of these paths is possible, thus we also evaluate the viability of shallow-land burial. This last path depends on the waste disposal rate index, which should be below unity to allow a SLB. Independently of the waste management strategy, we also evaluate how to handle the material, remote or hands-on manipulation. Mean the contact dose rate, we adopt a $10 \mu \mathrm{Sv} / \mathrm{h}$ limit for hands-on manipulation and assess the contact dose rates against this limit.

The computational methodology in this case consists in introducing the neutron fluxes calculated with MCNPX in the ACAB code to compute the following quantities: clearance index [13], Contact dose rate, specific activity, decay heat and waste disposal rating [15] as class $C$ waste.

\section{Results}

We present in this section the evaluation of the quantities mentioned in Section 2. After the presentation of the results, we assess their implications, and we explore different variations of the baseline design which could improve the found behavior.

\subsection{Burst operation}

During the operation, the neutron spread and the subsequent gamma production give rise to high dose rates that affect both the workers and the optical systems, and, when defined, to the electronic machinery and diagnostics. To evaluate the impact of the burst operation on the workers, we compute a dose rate map of the ambient dose equivalent. In this map it is also possible to find information about how the radiation is distributed in the target bay, and where are the most and least dose exposed locations in this design. We also compute the absorbed dose rate into the FOA to evaluate how important is the FOA Shield.

\subsubsection{Dose to workers}

In Fig. 3 there is the dose rate map of the ambient dose equivalent during the operation. It is assumed that a worker would stand in the 


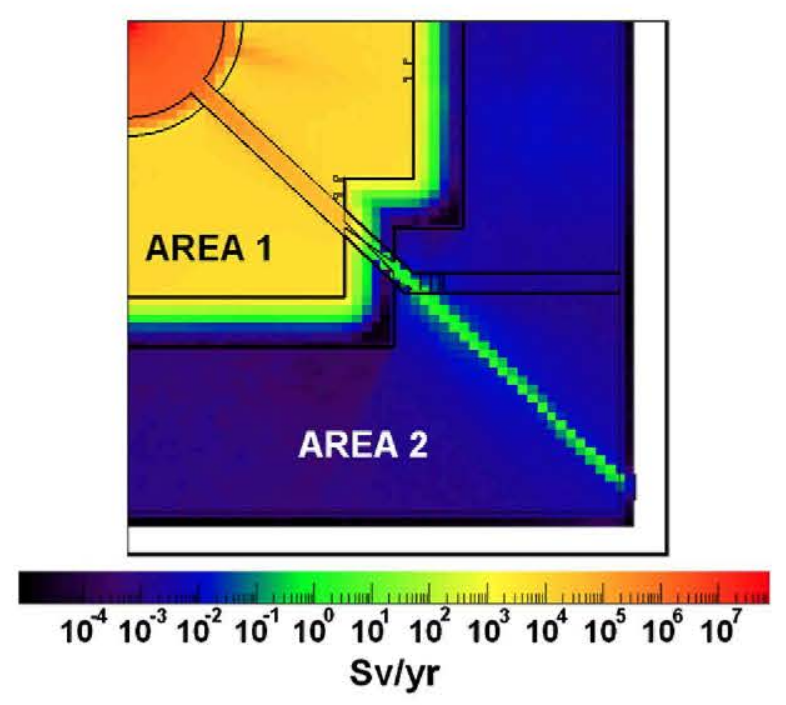

Fig. 3. Prompt dose rates maps assuming the presence of a person in all the explosions of the year. Ambient dose equivalent to workers. The Bioshield has been considered in the simulation.

facility during all the explosions taking place in a year. It is seen that the area 1 is well above the limit of $20 \mathrm{mSv} /$ year, thus workers are not allowed to stay there during the operation. The area 2 is mostly below the limit, but there are some locations which are above. The pinhole in the FOA shield represents a way out for the neutrons and gammas which projects a straight line of dose rate level above the limit.

In fact, the pinholes are the main responsible for the dose rates in the area 2. In Fig. 4, we have computed two dose rate maps. On the left side of Fig. 4, it is presented a dose rate map corresponding to the FOA shield with no penetrations. The dose rates are well below the limit for workers, and are close to $10^{-7} \mathrm{~Sv} /$ year. On the right side of Fig. 4, however, we have computed the resulting dose rate map exclusively due to the particles spreading through the pinholes; any particle coming out through other component is not considered for this calculation. The dose rates in any location of the area 2 (Fig. 3) are very close to those found in the right hand side of Fig. 4 . We conclude that the dose rates in area 2 are mainly due to the spreading of particles through the pinholes. We find unacceptable the simplification of no-pinhole FOA shield to assess the prompt dose rates, as there is a difference of up eight orders of magnitude. It is important

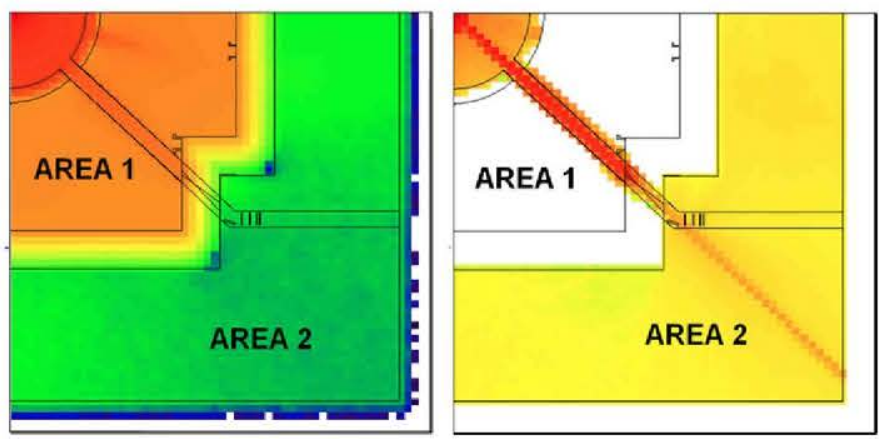

$$
\begin{gathered}
10^{18} 10^{15} 10^{14} 10^{13} 10^{12} 10^{11} 10^{10} 10^{9} 10^{8} 10^{7} 10^{-1} 10^{5} 10^{4} 10^{3} 10^{2} 10^{1} 10^{\circ} 10^{1} 10^{2} 10^{3} 10^{4} \\
\text { Sv/yr }
\end{gathered}
$$

Fig. 4. Left: prompt dose rates map due to all the particles except those crossing the pinhole. Right: prompt dose rates map due exclusively to the particles crossing the pinhole. Ambient dose equivalent to workers. The Bioshield has been considered in the simulation.
Table 1

Averaged absorbed dose rate in FOA for HiPER 4a advanced design and different contributions.

\begin{tabular}{lll}
\hline & Gy/year & Contribution \\
\hline Direct n & $5.18 \times 10^{-4}$ & $88.5 \%$ \\
Direct g & $6.67 \times 10^{-5}$ & $11.4 \%$ \\
Indirect n & $4.49 \times 10^{-10}$ & Negligible \\
Indirect g & $1.27 \times 10^{-7}$ & $0.1 \%$ \\
Total & $5.85 \times 10^{-4}$ & $100 \%$ \\
\hline
\end{tabular}

to note that the prompt dose rate may not be reduced by increasing the thickness if the FOA shield. There is a minimum dose rate during the burst operation in this design, which corresponds to the particles coming through the beam penetrations.

Obviously, this situation will be altered when roofs and floors were taken into account, as they will deflect the particles, and the dose rates in this area 2 will result more homogeneous and perhaps lower than the limit of $20 \mathrm{mSv} /$ year. However, two options could be explored to handle these locally high dose rates. The first one is the use of "dose stoppers" such as water tanks or polyethylene blocks placed in front of the pinholes outside the beam tubes. Other option could be a "dose tube", what is a tube made of a neutron reflecting material. Nothing would be inside these tubes, and dose rates outside would be attainable.

Considering the results of the previous design [1,2], together with the obtained results, we can conclude that outside the Bioshield, workers may stand during the operation of the facility. In effect, it was already a free-exclusion area in the previous design, and in the current design, there are bigger shields. Furthermore, it is seen in Fig. 3 that with the exception of the pinhole projections, the area 2 is close to the limit. Then, $2 \mathrm{~m}$ of extra shield, the Bioshield, will protect the workers.

\subsubsection{Dose to optics}

The optical systems can be deteriorated in the presence of neutron and gamma irradiation in several ways $[16,17]$. In the current design of HiPER 4a there are two groups of optical elements: the "renewable lenses" and the "Final Optics Assembly". The main threat for the renewable lenses is the short-term survivability. They are placed at $8 \mathrm{~m}$ from the center of the reaction chamber, and there is no shield between them and the particles and radiation resulting from the fusion explosions. Ions and X-rays will quickly melt and deteriorate the lenses. Some systems and special materials are being explored in the project to improve this situation, but nowadays, it is considered that damage in these lenses will be high enough to imply frequent renovations.

However, there is another group, the Final Optics Assembly, placed at $19 \mathrm{~m}$ from the center of the reaction chamber, which is composed by different optical components (mirrors, frequency convertors and focusing lenses). This group is very important and is protected in this scheme of Target Bay. The first protection, an indirect one, is the chamber shield, which reduces the amount of neutrons escaping from the chamber. The other two protections are direct shields devoted to decrease the damage in this group of lenses: (i) the Final Optics Assembly Shield, acting as a contention barrier limiting the end of area 1 and the beginning of area 2; and (ii) pinhole shield, decreasing the total amount of particles coming out through the penetrations in the FOA shield (see Fig. 2).

The damage generation in the lenses requires a study apart. However, we have assessed the average absorbed dose rates in all the components of the Final Optics Assembly to evaluate the impact of the shields on this group of lenses. In separate contributions, we depict in Table 1 the average absorbed dose rate derived exclusively from the particles spreading through the pinhole (direct particles) and, on the other hand, the averaged absorbed dose rates due to the rest of the particles (indirect particles). These contributions 
Table 2

Averaged absorbed dose rate in FOA for HiPER 4a advanced design with the absence of FOA shield and pinhole shield, and contributions.

\begin{tabular}{lllll}
\hline & $\begin{array}{l}\text { Neutrons absence } \\
\text { of shields }\end{array}$ & $\begin{array}{l}\text { Gamma absence of } \\
\text { shields }\end{array}$ & Total & Ratio \\
\hline Gy/year & 14.1 & 37.7 & 51.8 & 88547 \\
\hline
\end{tabular}

are subdivided in neutrons and gammas contributions. To assess the impact of the shields in the protection of the FOA, the results are compared, in Table 2, with the average absorbed dose rate in the absence of FOA and pinhole shields. The first point to note is the important reduction of absorbed dose rates in burst operation regime in the Final Optics Assembly compared to that in the previous design [1]. This is due to the reduction of total neutron yield $(120,000 \mathrm{MJ} /$ year in the previous irradiation scheme, $5200 \mathrm{MJ} /$ year in the current one) plus the increment of thickness in the chamber shield, FOA shield and pinhole shield.It is observed that absorbed dose rates arise mainly due to the particles coming out through the pinhole ( $99.9 \%$ of the total absorbed dose rate). Then, an increase of the FOA shield thickness cannot reduce the induced damage in this group of lenses. However, the presence of FOA and pinhole shield makes a considerable difference with the situation of absence of shields. The averaged absorbed dose rates are reduced by almost six orders of magnitude.

\subsection{Period between bursts}

Due to the neutron spreading during the explosions of the burst regime operation, the materials in the target bay get activated. The resulting decay gammas account for the residual dose rates which will affect both to workers and FOA.

There is no maintenance plan and no task definition to be performed in the target bay, thus we do not compute a dose rate map. Instead of it, we compute the time evolution of the averaged dose rates between bursts ( 1 week period). We also compute the contributions of the different components to the dose rates (both to workers and FOA) and its time evolution. In this way, we offer the following information: regarding to workers, the time evolution of the ambient dose equivalent averaged in whole areas is presented. Together with it, we also present the main contributors to the dose rates in that area, and its time evolution. With regards to FOA, we present the time evolution of the absorbed dose rates averaged in all the components and the time evolution of the main contributions to this quantity.

\subsubsection{Dose to workers from decay gammas}

In the period between bursts, certain tasks might be done in the target bay. The residual dose rates will impose the necessity of remote handling in some areas of the target bay, as manual handling will be possible in some others. We evaluate this situation by means of computing the averaged ambient dose equivalents in the following areas: inside the reaction chamber, and areas 1, 2. Considering the study in the previous design, we conclude that area 3 does not present any trouble related to activation, and no further study is made here.

In Fig. 5, we present the time evolution of the averaged residual dose rate from the end of a burst up to the beginning of the next one (one week of period between bursts) for the different rooms. As it is concerned to workers, we calculated the ambient dose equivalent. It has been found no accumulative effect between bursts. In the period of one week, the dominating isotopes to the residual dose rate are short-life isotopes. The information that we present, is referred to the period after 30 years of irradiation, but we have verified that it is the same that after the first burst.

\section{Residual dose rate to people between bursts}

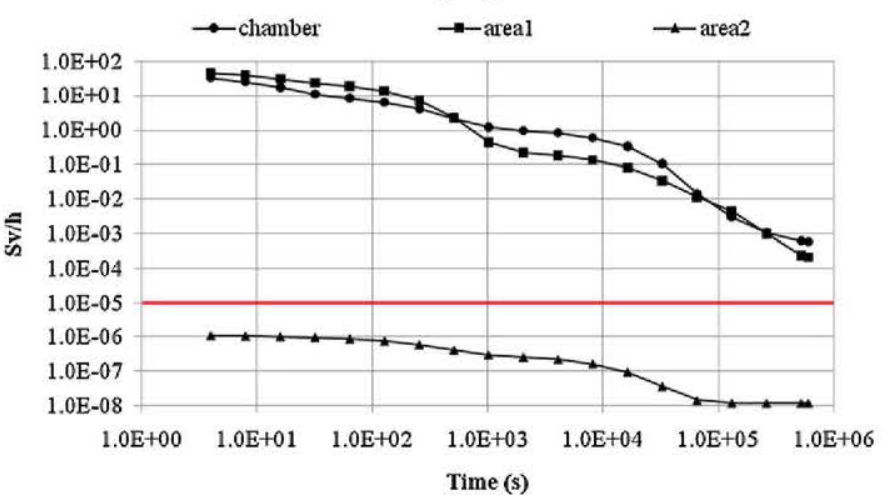

Fig. 5. Averaged residual dose rate between bursts to workers inside the chamber, in area 1 and area 2 . Ambient dose equivalent to workers after the last burst.

Inside the chamber and in area 1, remote handling is necessary, as the limit of $10 \mu \mathrm{Sv} / \mathrm{h}$ is not fulfilled between bursts. This behavior is not altered from the previous design. However, in area 2, manual maintenance is possible from the end of the burst. In the previous design, it was necessary to spend $36 \mathrm{~h}$ of cooling time before the limit was reached. Then, there is no motivation for exploring alternatives to reduce the residual dose rate in area 2 . This change is due to the increase of shields and the reduction of total neutron yield, as the amount of neutrons reaching this area 2 has decreased significantly.

We go deeper in the analysis of the situations inside the chamber and area 1, as they are the troublesome rooms in the period between bursts. Inside the chamber, the contributions to the residual dose rate are found in Fig. 6 .

From the shutdown to $1 \mathrm{~h}$, the chamber and the rims contributions increase from a $15 \%$ to $95 \%$, decreasing the contribution of the chamber shield. This is also their contribution at the time of one week, when the next burst starts. The residual dose rates arising from the chamber and rims at the time of one week are mainly due to ${ }^{106} \mathrm{Ag}^{\mathrm{m}}$ (7.8\%), ${ }^{110} \mathrm{Ag}^{\mathrm{m}}$ (25.7\%) and ${ }^{54} \mathrm{Mn}$ (46.2\%). The two first isotopes derive from the $\mathrm{Ag}$ initial concentration in the T91 steel alloy, being an impurity. The ${ }^{54} \mathrm{Mn}$ derives from the Fe, main intended element of the steel alloy.

In the area 1, the contributions of the different components to the residual dose rate are depicted in Fig. 7.

The pinhole shield and FOA shield dominate $(90 \%$ of the total contribution) from the shutdown to one hour. After one hour, the situation is inverted ( $<30 \%$ of the total contribution) in benefit of the tubes and rails. At one week, the main contributions are in

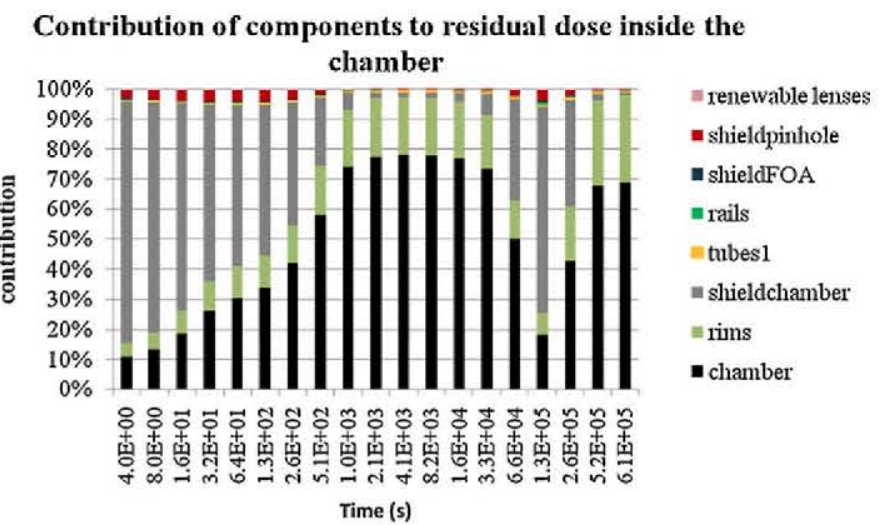

Fig. 6. Contribution of target bay components to residual dose rates inside the chamber. Ambient dose equivalent to workers. 
Table 3

Main contributing isotopes to residual dose rate in area 1 at one week.

\begin{tabular}{|c|c|c|}
\hline Component & Contribution & Main isotopes \\
\hline Rims & $4 \%$ & ${ }^{106} \mathrm{Ag}^{\mathrm{m}}(4.9 \%),{ }^{110} \mathrm{Ag}^{\mathrm{m}}(41.8 \%),{ }^{54} \mathrm{Mn}(32.1 \%)$ \\
\hline Rails & $5 \%$ & ${ }^{58} \mathrm{Co}(10.4 \%),{ }^{60} \mathrm{Co}(29.7 \%),{ }^{59} \mathrm{Fe}(26.8 \%),{ }^{54} \mathrm{Mn}(26.5 \%)$ \\
\hline Pinhole Shield & $25 \%$ & ${ }^{46} \mathrm{Sc}(94.6 \%),{ }^{24} \mathrm{Na}(5.0 \%)$ \\
\hline Tubes 1 & $27 \%$ & ${ }^{106} \mathrm{Ag}^{\mathrm{m}}(9.4 \%),{ }^{110} \mathrm{Ag}^{\mathrm{m}}(18.6 \%),{ }^{54} \mathrm{Mn}(49.9 \%)$ \\
\hline FOA shield & $40 \%$ & ${ }^{46} \mathrm{Sc}(83.3 \%),{ }^{24} \mathrm{Na}(9.1 \%)$ \\
\hline
\end{tabular}

Table 3.Given the specific contribution of the components, the most important isotopes at that time in the area 1 are ${ }^{46} \mathrm{Sc}$ and ${ }^{54} \mathrm{Mn}$. ${ }^{46} \mathrm{Sc}$ derives from $\mathrm{Sc}$ in the concrete; ${ }^{54} \mathrm{Mn}$ is produced in the $\mathrm{Fe}$ of the T91 steels alloy of the tubes. Replacing the materials for the tubes or exploring the viability of zero presence of Scandium in the concrete could result in the reduction of the dose rates. These changes represent a factor 9 of reduction of the dose rates, they would be enough, as a reduction factor 24 is necessary to allow manual handling in area 1 before 1 week.If manual maintenance is mandatory in this area 1 , further strategies will be necessary. We would suggest, timetable planning for exposures, different shielding configurations and alternative materials for FOA and pinhole shielding.

\subsubsection{Dose to optics from decay gammas}

The FOA is a very important group of optical elements. This group focuses the laser beam, it converts its frequency, and extracts a part of it to do diagnostics. It is then important that the damage due to the irradiation does not alter its optical properties.

During the burst operation, a combined irradiation of neutrons and gammas reaches this group of lenses during very short periods of time. In the period between bursts, this group is exposed to continuous gamma irradiation, much lower in intensity, but for significantly longer times of exposures. The integrated deposited energy over a year could give rise to a source of damage similar to that of burst operation. Thus, we compute the absorbed dose rate during the period between bursts.

In Fig. 8, we show the time evolution of the residual dose rate in the FOA. It is seen that integrating the dose rates over the whole year, this term of dose is in the order of magnitude of $10^{-3} \mathrm{~Gy} /$ year, what is higher than the integrated dose rate deposited in a year due to burst operation. Thus, if the absorbed dose rates during the burst operation (neutron and gammas, Table 1) were considered to be troublesome, the integrated residual dose rate (only gamma) also has to be taken into account as a source of damage, as they are similar in magnitude.

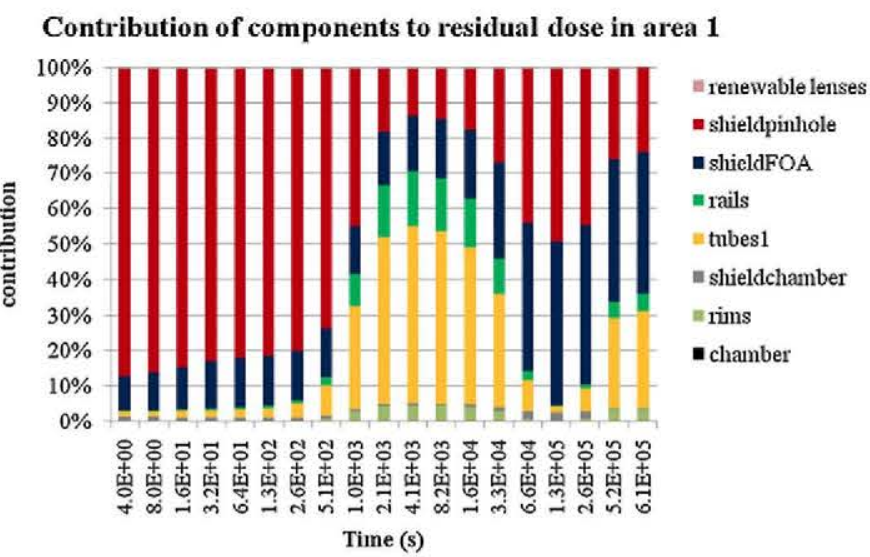

Fig. 7. Contribution of target bay components to residual dose rates in area 1 . Ambi ent dose equivalent to workers.

\subsection{End of life}

The estimated lifetime for the facility HiPER $4 a$ is 30 years. The waste management of the facility after the irradiation is mandatory from the design phase, to minimize the volume, and plan the decommissioning. We focus on strategies that avoid the burial or long-term storage. Thus, we explore the two following strategies: clearance and recycling. We also compute, as a guide the WDR index, to know if the waste could be allocated in a shallow-land burial, as last alternative if clearance and recycling were not possible. The quantities are evaluated for activated materials after 30 years of irradiation.

To perform the evaluation of clearance, we follow the clearance index. If this $\mathrm{CI}$ index is below the unit, the clearance of this material is possible. This option is the preferred solution, as the components would undergo declassification as radioactive material. However this strategy is very demanding and it is not always possible. Regarding to recycling, we consider the procedures given in [14]. If the material to be recycled is steel, it is necessary to reach a specific activity below $1000 \mathrm{~Bq} / \mathrm{cm}^{3}$; this permits to process the steel alloy in foundries. If it is not steel, the only restriction for recycling has to do with the decay heat. Below $2000 \mathrm{~W} / \mathrm{m}^{3}$ [14], active cooling is no longer necessary, and recycling is possible. Furthermore, the evaluation of the contact dose rate (CDR) indicates whether the material may be manually handled or if remote handling is necessary for the manipulation.

In Table 4 we present the evaluation of the different indexes for all the components of the target bay. The appearing order in the table is done by materials: steels, concretes and silica. The steel selection for the reaction chamber is deeply studied in [2].

In general, the components made of steel do not easily fulfill clearance limits (with the exception tubes in area 2), and they require less than 30 years to be recycled in foundries and less than 20 years to allow manual handling. The components made of concrete can undergo clearance with few years of cooling time (with the exception of the chamber shield, which requires 70 years). They can be recycled from the shutdown, given their low residual heat, and require some months to allow the manual handling. The optical components, which will not be probably exposed to radiation

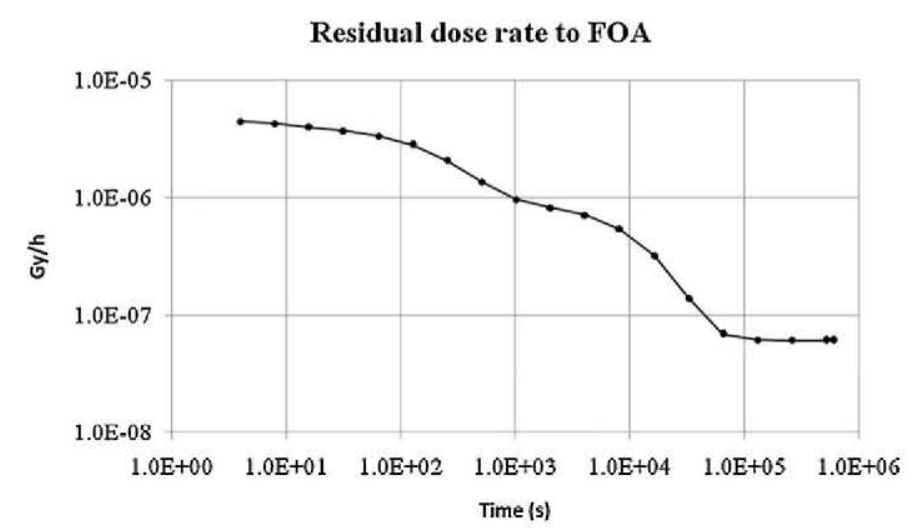

Fig. 8. Residual dose rate to FOA. Averaged absorbed dose rate in all the components. 
Table 4

Waste management assessment summary for the target bay components.

\begin{tabular}{llll}
\hline Component & Clearance & Recycling & Manual handling \\
\hline Chamber & No & 26 years & 20 years \\
Rims & No & 20 years & 15 years \\
Tubes1 & 45 years & 15 years & 5 years \\
Tubes2 & Always & Always & $0.5 \mathrm{~h}$ \\
Rails & 30 years & 5 months & 2 weeks \\
Chamber shield & 70 years & Always & 3 months \\
Pinhole shield & 5 years & Always & 1 month \\
FOA shield & 3 years & Always & 6 months \\
Bio shield & Always & Always & Always \\
Renewable lenses & 3 days & Always & $2 \mathrm{~h}$ \\
FOA & Always & Always & $0.5 \mathrm{~h}$ \\
\hline
\end{tabular}

the 30 years of lifetime of the facility, can undergo clearance and be recycled manually from almost the shutdown.We have also evaluated the WDR for Shallow-land burial (SLB) as Class C wastes [15]. It is not necessary because the components can be managed with no radiological waste disposal (clearance and recycling), but we estimated important to offer this information for comparing with DEMO reactors, were SLB is an important alternative. We found that the WDR is orders of magnitude below the limit for SLB thus the whole target bay could be allocated in a shallow-land burial if necessary.There is however another issue in connection with the WMA, related to the residual dose rate emitted by all the components. It is critical to decide if remote operation is necessary to extract the components from the target bay. Certain tasks of pre-processing for the waste management (cutting, packing, cleaning. . .) might be done in situ. We have then computed in Fig. 9, the time evolution of the residual dose rate inside the reaction chamber and in area 1 from 1 week to 100 years, together with the time evolution of the contributions of the components.

It is necessary to spend 3 years to allow the entrance in the area 1 , and 7 years to allow the entrance inside the chamber. After this time, and fulfilling the restrictions imposed by the CDR of the components inside the rooms (Table 4), manual manipulation is allowed. This option is an advantage because it avoids the necessity of certain equipment to prepare the waste management of the components.

The contributions of the different components to the residual dose rates inside the chamber are the same from 1 week to 7 years (see Fig. 6). Inside the chamber the responsible components for the dose rates are the chamber itself and the rims.

In the area 1 , the time evolution of the contributions from 1 week to 4 years is shown in Fig. 10.

It is seen that from 6 months to 4 years, the tubes are the main responsible for the residual dose rate in area 1 . Around 4 years, the

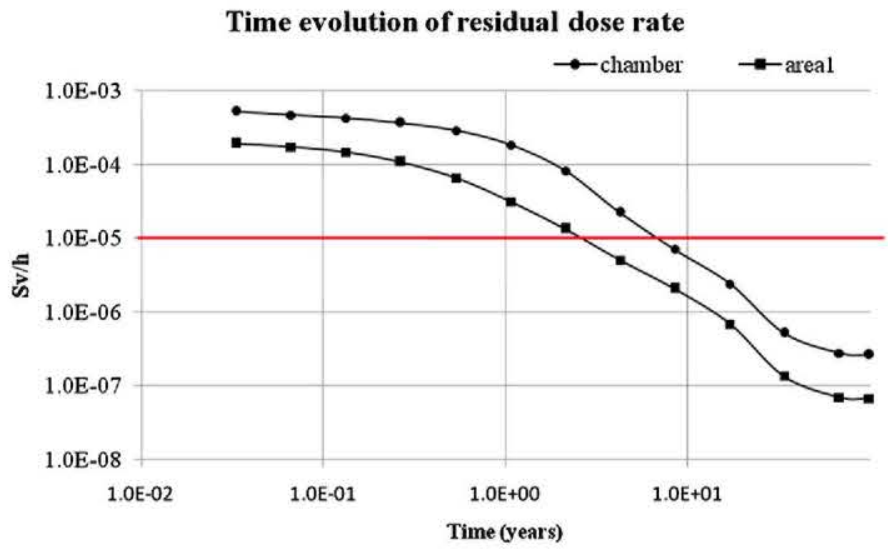

Fig. 9. Averaged residual dose rate after the final shutdown inside the chamber and in area 1 . Ambient dose equivalent to workers.

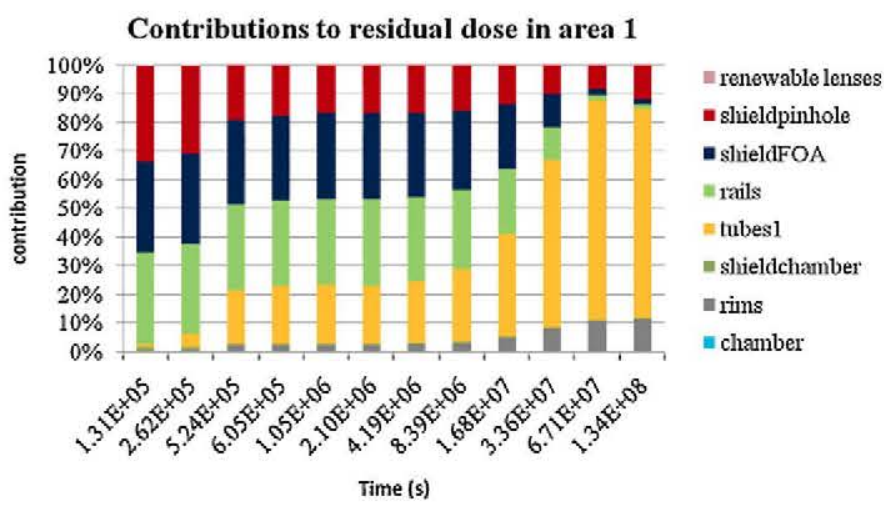

Fig. 10. Contribution of target bay components to the residual dose rate after the final shutdown in area 1.

main isotopes contributing to it are ${ }^{60} \mathrm{Co}$ and ${ }^{54} \mathrm{Mn}$. The first one derives from both the $\mathrm{Ni}$ and $\mathrm{Co}$ impurities in T91, and the second one derives from the Iron. Impurities control could improve the situation reducing the dose rate at that time. Other strategy would be replacing the tubes and rails material; the cooling time to allow the entrance in area 1 could be shorter by means of lower activation materials for tubes 1 and rails as they account for the $55 \%$ of the contribution to the dose rates around 4 years of cooling time.

\section{Conclusions}

We have performed an overview of the radiological response of the facility divided in three stages: burst operation, period between bursts and decommissioning. The HiPER 4a model is divided in rooms occupying the space between shields: between the chamber shield and the FOA shield we find the area 1. Outside the FOA shield and inside the Bioshield the area 2 is found. The space outside the Bioshield is the area 3.

In the burst operation, the entrance in area 1 is forbidden. In area 2 there are some places where workers could stand, but it is recommended a deeper analysis, taking into account future details definitions in the design such as roofs, floors, doors, equipment penetrations, etc. In area 3 the entrance is allowed with no exception. Regarding to the computation of ambient dose equivalent, it has been found that more than $99.99 \%$ of the doses to workers in any location of area 2 are due to particles coming through the pinholes. It is has been found unacceptable the simplification in the modeling of a no-pinhole FOA shield. With regards to the final optics assembly, in this new design, it has been found that the absorbed dose rates have significantly decreased compared to the early design, given the increase in the chamber shield, FOA shield, and pinhole shield thickness. The $99.9 \%$ of the dose rates in FOA are due to particles coming through the pinhole. It has also been assessed the impact of the presence of the FOA shield and pinhole shield in the FOA absorbed dose rate. It has been estimated that the presence of these shields reduces into almost six orders of magnitude the absorbed dose rate in FOA.

The calculation of ambient dose equivalent in the period between bursts indicates that the entrance is forbidden inside the chamber and in the area 1 . The main responsible components of the target bay for the residual dose rates inside the chamber and area 1 have been identified together with the most contributing isotopes. Inside the chamber at one-week, the chamber and rims are the responsible components for the dose rates, and ${ }^{106} \mathrm{Ag}^{\mathrm{m}},{ }^{110} \mathrm{Ag}^{\mathrm{m}}$ and ${ }^{54} \mathrm{Mn}$ the responsible isotopes. The two first derive from the $\mathrm{Ag}$ in the T91, an impurity. The last one derives from the Fe, main intended element is the steel alloy. In the area two, the responsible components for the dose rates ate one week are the tubes 1 , the 
pinhole shield and the FOA shield. The main responsible isotopes are ${ }^{54} \mathrm{Mn}$ and ${ }^{46} \mathrm{Sc}$. The first one derives from the Fe of the tubes steel T91, and the second one is created from the Sc present in the concrete of the shields. The area 2 and the area 3 are free of restrictions from the end of the burst. In the previous design, the area 2 needed $36 \mathrm{~h}$ to allow the entrance, but the increment of shields thicknesses changed this situation. With regards to absorbed dose rates in FOA in the period between bursts, it is found that the yearintegrated residual dose rate and the year-integrated prompt dose rate in the FOA are of the same order of magnitude.

The waste management evaluation shows attractive alternatives in the short and mid-term. All the components, with the exception of the chamber, the rims, the chamber shield and the tubes of area 1 , fulfill the clearance limits before 30 years. Those components which cannot undergo a clearance in 30 years may be manually recycled before 30 years. Thus, we conclude that in 30 years, the whole target bay may be managed as a waste with no burial needs. We have also evaluated the residual dose rate inside the chamber and in area 1. This information is important to make decisions on the kind (remote or manual) of in situ pre-processing (cutting, packing, cleaning. . .) for the waste management of the components. It has been found that after 3 years, the entrance is allowed in area 1 , while 7 years are necessary to access the interior of the reaction chamber. The results have been obtained considering impurities in commercial materials. This means that the impurities effect is not very relevant, since its presence does not imply advanced control to allow a reasonable waste management.

\section{Future work}

In future work, it is necessary to study in detail the response of the FOA, element by element. The impact of the dose coming through the pinhole might be critical in the facing mirror. Furthermore, if the ions and X-rays impact on the renewable lenses is avoided, and it is assumed a long-term survivability for these components, it will be necessary to evaluate the absorbed dose induced by neutrons and gammas in this group of lenses.

When a plan of maintenance is defined, it will be interesting to compute a residual dose rate map. Together with the time-table information, it can be calculated the collective dose to workers, and support the decision making on maintenance plan.

Further details definitions in the design will alter the obtained results. The presence of floors and roofs, doors, and new equipment penetrations will modify the prompt and the residual dose rates and the WMA. The presence of machinery, properly shielded against radiation, will also alter the residual dose rates. In this HiPER 4a design, no component with structural function has been defined. When the brackets, supports and concrete structure will be defined, some additional amount of steel will be probably introduced in the target bay, and another radiological characterization will have to be accomplished.

\section{Acknowledgments}

The authors gratefully acknowledge the support of HiPER: European High Power Laser Energy Research Facility (Preparatory phase study). Fp7-infraestructure-2007-1.VII Framework Program, Capacities workprogram, grant agreement no. 211737.

It has been also partially supported by Plan Nacional I+D+I (2008-2011) Fusion Nuclear ENE2008-06403-C06-02 MICINN (Spain) and the European Union keep-in-touch Program on IFE.

R. Juárez, J. Sanz, J.M. Perlado, B. Le Garrec, Dose rates evaluation of HiPER facility, Fusion Eng. Des. 86 (2011) 694-698.

J.Sanz, R. Juárez, F. Ognissanto, J.M. Perlado, Waste management assessment of candidate materials for HiPER react ion chamber, Fusion Sci. Technol. 60(2011) $579-584$

R.L. Klueh, E.T. Cheng, M.L. Grossbeck, E.E. Bloom, Impurity effects on reducedactivation ferritic steels developed for fusion applications, J. Nucl. Mater. 280 (2000) 353-359.

J.F. Latkowski, Measurement of the NIF gunite shielding composition and implications for neutron activation and workers doses, Fusion Technol. 39 (2001) 956-959.

Stephen Sanders private communication. Oxford Technologies. stephen.sanders@oxfordtechnologies.co.uk.

ICRP publication 74: Conversion Coefficients for use in Radiological Protection against External Radiation, Pergamon Press (1997)

ICRP publication 60, Annals of the ICRP, vol, 21, no. 1-3 (1990).

Y. Wu, FDS Team 2009, Fusion Eng. Des. 84 (2009) 1987-1992

D.B. Pelowitz, MCNPX User's Manual and extensions, LA-CP-05-0369 (2005)

M.B. Chadwick, P. Oblozinsky, M. Herman, et al., ENDF/B-VII.0: next generation evaluated nuclear data library for nuclear science and technology, Nucl. Data Sheets 107 (2006) 2937-3060.

J-Ch Sublet, LW Packer, J. Kopecky, R. A Forrest, AJ Koning D A Rochmand, The European Activation File: EAF-2010 neutron-induced cross section libraries Culham Science Centre, Rept. CCFE-R(10) 05 (2010)

I. Sanz, O. Cabellos, N. Garcíaherranz, ACAB-2008: Inventory code for nuclear applications, Nuclear Energy Agency NEA Data Bank, NEA-1839 (2008)

L.W. Packer, J.-Ch. Sublet, The European Activation File: EAF-2010 biological, Clearance and transport libraries Culham Science Centre, Rept, CCFE-R(10) 04 (2010).

M. Zuchetti, L. Di Pace, L. El-Guebaly, B.N. Kolbasov, V. Massaut, R. Pampin, et al., An integrated approach to the back-end of the fusion materials cycle, Fusion Eng. Des. 83 (2008) 1706-1709.

S. Fetter, E.T. Cheng, F.M. Mann, Long term radioactive waste from fusion reactors: part II, Fusion Eng. Des. 13 (1990) 239-246.

C.D. Marshall, J.A. Speth, S.A. Payne, Induced optical absorption in gamma, neutron and ultraviolet irradiated fused quartz and silica, J. Non-Crystal. Solids 212 (1997) 59-73.

J.F. Latkowski, A. Kubota, M.J. Caturla, S.N. Dixit, J.A. Speth, S.A. Payne, Fused silica final optics for inertial fusion energy: radiation studies and system-leve analysis, Fusion Sci. Technol. 43 (2003) 540-558. 